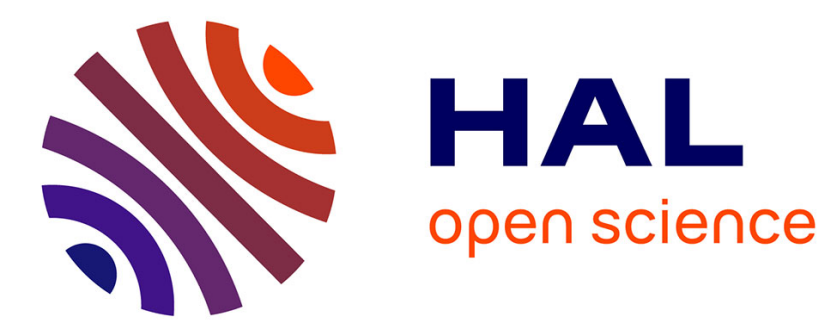

\title{
L'énergie dans la région baltique, un enjeu primordial de sécurité
}

Céline Bayou

\section{To cite this version:}

Céline Bayou. L'énergie dans la région baltique, un enjeu primordial de sécurité. Stratégique, 2020. hal-03286359

\section{HAL Id: hal-03286359 \\ https://hal.science/hal-03286359}

Submitted on 14 Jul 2021

HAL is a multi-disciplinary open access archive for the deposit and dissemination of scientific research documents, whether they are published or not. The documents may come from teaching and research institutions in France or abroad, or from public or private research centers.
L'archive ouverte pluridisciplinaire HAL, est destinée au dépôt et à la diffusion de documents scientifiques de niveau recherche, publiés ou non, émanant des établissements d'enseignement et de recherche français ou étrangers, des laboratoires publics ou privés. 


\section{L'énergie dans la région baltique, un enjeu primordial de sécurité}

\section{Céline Bayou*}

En proie aux injonctions contemporaines concernant la transition énergétique, les pays de la région de la mer Baltique doivent, comme d'autres, procéder à la réévaluation de leurs politiques énergétiques nationales: décarbonisation, montée en puissance des énergies renouvelables (EnR) mais aussi du gaz naturel liquéfié (GNL), positionnement concernant l'énergie nucléaire mais aussi diversification des mix de production et de consommation, ainsi que des fournisseurs et des voies d'approvisionnement sont à l'ordre du jour pour tous les pays.

Dans la région baltique, il convient d'ajouter à ces préoccupations partagées celles, spécifiques mais tout aussi urgentes et impérieuses, d'une zone qui s'est trouvée 50 ans durant à la jonction des blocs de l'Est et de l'Ouest et reste caractérisée par le déséquilibre des acteurs en termes de dotation énergétique. Il en découle une organisation systémique des réseaux énergétiques, tout particulièrement dans les secteurs électrique et gazier.

Ces interdépendances rémanentes sont perçues par certains pays comme des facteurs accroissant les menaces qui pèsent sur eux et la région. Afin de gérer ce qu'ils perçoivent comme un risque, ils s'efforcent de procéder à une modification radicale des interconnexions énergétiques. Depuis le début des années 1990, les pays de la rive sud de la Baltique en particulier - de l'Estonie à la Pologne, en passant par la Lettonie et la Lituanie - travaillent à se détacher du pôle ex-soviétique et à s'insérer pleinement dans celui d'Europe occidentale, processus de réorientation coûteux et techniquement complexe, mais soutenu par l'Union européenne (UE). La réalisation de cette réorientation radicale se heurte pourtant à de nombreux obstacles, qui font parfois douter de la volonté politique réelle des acteurs de la région. Outre le fait que l'objectif de déconnexion du fournisseur russe d'hydrocarbures ou de technologies est entravé par une proximité géographique et historique objective, les positions de l'UE ou de certains de ses États membres portent parfois la marque de la volatilité. Par ailleurs, les concurrences à l'œuvre sur le plan régional retardent, voire annihilent, certains projets, quitte à perdre en efficacité. Finalement, il n'est pas rare que les décisions adoptées au niveau national, généralement justifiées par des objectifs communs, se trouvent en

\footnotetext{
* Chargée de cours à l'Institut national des langues et civilisations orientales (INALCO) et à l'IRIS (Institut des relations internationales et stratégiques), Rédactrice en chef du site Regard sur l'Est.
} 
contradiction avec ces derniers. Les concurrences sont-elles si fortes qu'elles puissent mettre à bas des intérêts régionaux et sub-régionaux pourtant bien compris ? Près de trente ans après avoir été annoncée, la déconnexion/reconnexion des pays de la région s'en trouve en tout cas retardée. Et, par l'importance qu'il a acquise en termes économiques et politiques, le champ énergétique apparaît désormais comme un terrain de confrontation majeure entre les acteurs intervenant dans la région baltique.

\section{Des transitions énergétiques totales}

Quelles qu'en soient les justifications, des transitions énergétiques sont à l'œuvre dans l'ensemble des pays de la région de la mer Baltique, comme ailleurs dans le monde. Le Danemark, par exemple, peut désormais se prévaloir de produire 47,7\% de son électricité grâce aux EnR et annoncer de manière crédible qu'à horizon 2050 le pays, déjà situé au premier rang européen pour la production d'électricité éolienne, sera libéré des hydrocarbures. Il en va presque de même pour la Suède qui, en 2015, produisait déjà $64 \%$ de son électricité grâce aux EnR (hydroélectricité, éolien et biomasse) et s'est engagée à passer à $100 \%$ d'EnR en 2040. Peu après la catastrophe de Fukushima (2011), l'Allemagne a décrété l'arrêt définitif de la totalité de ses centrales nucléaires au plus tard en 2022. La Pologne, sous l'effet des régulations européennes et internationales en matière d'émissions de gaz à effet de serre, va devoir procéder à des modifications importantes de ses structures énergétiques, alors que $80 \%$ de sa production d'électricité venait encore du charbon et du lignite en $2017^{1}$.

Aux politiques de lutte contre le changement climatique ou au traumatisme provoqué par 1'accident nucléaire de 2011, il faut ajouter la révolution du gaz de schiste qui a rebattu les cartes des marchés traditionnels d'exportation et d'importation, la volatilité des prix des hydrocarbures, la part croissante du GNL et les innovations technologiques.

À ces contraintes ou opportunités nouvelles s'ajoutent pour certains pays baltiques des spécificités liées à leur proximité géographique et/ou historique de la Russie. La problématique est double pour eux : il s'agit à la fois de réduire leur dépendance vis-à-vis d'un partenaire désormais jugé peu fiable et menaçant, et de s'adapter à la volonté de ce dernier de réduire

\footnotetext{
${ }^{1}$ Kai-Olaf Lang, « Energy Security in the Baltic Sea Region : EU Members of the Region between Integration and Discord », in Māris Andžāns, Ilvija Bruğe (dir.), The Baltic Sea Region : Hard and Soft Security Reconsidered, Latvian Institute of International Affairs, Riga, 2016. En décembre 2019, Varsovie a toutefois obtenu un délai supplémentaire avant de se conformer aux engagements communautaires de neutralité carbone à horizon 2050.
} 
leur rôle en tant que pays de transit d'hydrocarbures. L'Estonie, la Lettonie, la Lituanie, mais aussi la Finlande et la Pologne, sont tout particulièrement concernées par ces aspects qui les touchent en tant que pays membres de l'UE mais aussi en tant que pays frontaliers ou très proches de la Russie, le cas extrême étant constitué par les pays baltes, anciennement membres de l'Union soviétique. Parties intégrantes, à ce titre, du système énergétique soviétique jusqu'en 1991, ils incarnent aujourd'hui l'exemple type de cette «insularité énergétique » parfois évoquée à propos des pays de la région ${ }^{2}$. Si des connexions ont été réalisées depuis le recouvrement des indépendances baltes - la Finlande est également concernée par cette dynamique et est souvent assimilée au groupe de ces îles énergétiques de la Baltique -, la dépendance de ces pays vis-à-vis de la Russie reste grande, et ce malgré la volonté de l'UE de les intégrer dans un système européen plus large.

\section{Quand l'UE fixe les grandes lignes}

Le Plan d'interconnexion des marchés énergétiques de la région de la Baltique (PIMERB) résulte d'un accord signé en 2009 à l'initiative de la Commission européenne avec les huit pays de la région (Allemagne, Danemark, Pologne, Estonie, Lettonie, Lituanie, Finlande, Suède $)^{3}$ et visant à mieux connecter les trois Baltes aux réseaux énergétiques de l'UE. Il s'agit donc de créer un marché énergétique intégré, appuyé par des infrastructures qui contribueront à renforcer la sécurité énergétique de l'ensemble de la région.

Le PIMERB intervient essentiellement dans le domaine des interconnexions électriques, afin de renforcer les réseaux entre les trois pays baltes, entre eux et leurs voisins nordiques et, enfin entre la Pologne et l'Allemagne. Le Plan intervient également, plus discrètement, dans le domaine du marché intérieur du gaz et des infrastructures afférentes. Il s'agit là d'améliorer la sécurité d'approvisionnement en visant une plus grande diversification des sources et des routes, ce qui suppose des interconnexions nouvelles, la mise en place d'infrastructures permettant l'inversion des flux, des installations de GNL et le développement du stockage de gaz.

Outre la promotion de marchés intégrés de l'électricité et du gaz ainsi que la sécurité d'approvisionnement, le développement des infrastructures, celui de la production ou

\footnotetext{
${ }^{2}$ Greta Tučkutè, « Challenges and Opportunities in Nordic-Baltic Energy Connectivity - Turning an Energy Island into an Integrated Part of the European Energy System », in Kinga Redłowska (ed.), Baltic Visions European Cooperation Region Stability, Foundation Institute for Eastern Studies, Varsovie, 2015, pp. 23-31.

${ }^{3}$ La Norvège en est observateur.
} 
l'accroissement de l'efficacité énergétique, le PIMERB promeut aussi les EnR (notamment l'éolien) en soutenant certains projets précis ${ }^{4}$. Parce qu'il vise à créer une union de l'énergie en s'attachant à promouvoir la coopération régionale, le PIMERB est considéré au sein de l'UE comme un exemple de « bonne pratique ».

\section{Les interconnexions électriques en bonne voie}

La synchronisation des réseaux n'est pas chose facile. L'insuffisance des infrastructures et les différences techniques constatées entre les installations nécessitent des investissements conséquents. Il faut donc que la volonté politique soit suffisante pour les assurer.

Au début des années 1990, les réseaux électriques des pays baltes étaient exclusivement synchronisés avec ceux de la Russie et de la Biélorussie, dans le cadre de l'accord BRELL (Belarus, Russia, Estonia, Latvia, Lithuania). Les trois Baltes sont aujourd'hui à cheval entre plusieurs systèmes, la Russie continuant à contrôler et équilibrer le réseau BRELL, dont elle tente par ailleurs d'assurer le maintien ${ }^{5}$. Les Baltes se fixent comme objectif ultime une déconnexion totale de ce système et l'horizon, fixé à 2025, est désormais visible.

Le fait que ces trois pays se trouvent dans des situations très différentes au regard de la production d'électricité favorise chez eux des politiques elles aussi diverses, les priorités de l'un n'étant pas celles de l'autre. Ainsi, l'Estonie exploite les schistes bitumineux présents sur son territoire, qu'elle transforme en électricité (lui permettant d'assurer jusqu'à $80 \%$ de sa production d'électricité) ou en huile de schiste, et qui font d'elle l'un des pays d'Europe les moins dépendants des sources extérieures d'énergie (à peine $12 \%$ ). Elle s'est par ailleurs lancée dans une politique active en faveur des EnR (biomasse et éolien) qui peuvent contribuer déjà à $14 \%$ de sa production d'électricité et doivent atteindre $50 \%$ d'ici 2030 . L'Estonie est actuellement exportatrice nette d'électricité, notamment vers la Finlande, la Lettonie et la Lituanie. La Lettonie, de son côté, produit la plus grosse part de l'électricité qu'elle consomme grâce aux centrales hydroélectriques installées durant la période soviétique le long de la Daugava. Elle est d'ailleurs l'un des pays européens les plus avancés en matière de renouvelable qui, certaines années, contribue à plus de $68 \%$ de sa production d'électricité. La Lituanie, enfin, était un exportateur net d'électricité jusqu'en 2009 et la fermeture, imposée par l'UE, de la centrale nucléaire d'Ignalina. Depuis, elle est le plus vulnérable des trois pays

\footnotetext{
${ }^{4}$ Voir notamment Baltic Energy Market Interconnection Plan, Action Plan, 2009.

${ }^{5}$ Pour la Russie, l'abandon du réseau BRELL poserait des difficultés d'alimentation électrique de l'enclave de Kaliningrad.
} 
pour ce qui concerne le risque de rupture d'offre d'électricité. Elle est devenue importateur net, grâce à l'électricité fournie par l'Estonie, la Lettonie et les pays nordiques depuis l'installation de câbles sous-marins. Mais elle importe aussi désormais de l'électricité de Russie, ce qu'elle perçoit comme une fragilité supplémentaire. De ces situations spécifiques découlent des priorités diversement formulées, qui ne favorisent pas forcément la mise en œuvre d'une politique commune en matière de raccordement.

Des interconnexions sont toutefois en cours, essentiellement avec les pays nordiques et avec la Pologne. Il s'agit généralement de câbles de capacité modeste, négociés le plus souvent de façon bilatérale, avec participation de l'UE mais sans approche globale préalable. Les liens qui se tissent se concrétisent peu à peu et ne répondent pas à l'établissement d'un réseau préétabli visant à quadriller l'ensemble de la région de façon la plus rationnelle possible.

Ainsi, à la fin de 2006 puis en mars 2014 ont été inaugurés les câbles Estlink 1 et Estlink 2, d'une capacité respective de 350 et $650 \mathrm{MW}$ par an, reliant 1'Estonie et la Finlande. Ces interconnexions sont gérées conjointement par les opérateurs des deux pays, Elering et Fingrid, mais la Lettonie, la Lituanie et l'UE ont participé à leur financement. Un autre câble sous-marin relie depuis fin 2015 la Suède et la Lituanie : baptisé NordBalt, il a rencontré de nombreux obstacles, en phase préparatoire mais aussi au moment de sa pose au fond de la Baltique (la Flotte russe est venue à plusieurs reprises perturber son installation en pénétrant dans les eaux territoriales lituaniennes, forçant les navires assurant la pose du câble à détourner leur course). Le câble est doté d'une capacité maximale de $700 \mathrm{MW}$. Un autre câble a été installé fin 2015, entre la Lituanie et la Pologne cette fois : le LitPol Link sera doté à terme d'une capacité de $1000 \mathrm{MW}$ par an. Tous ces projets ont bénéficié du soutien financier de l'UE, dans le cadre du PIMERB, et sont considérés comme les premiers pas vers la synchronisation du réseau électrique baltique avec le reste de l'Europe et vers la désynchronisation du réseau BRELL. À partir de 2016, l'avancée de ces projets a porté certains commentateurs à choisir de ne plus évoquer des «îles énergétiques » à propos des pays de cette région, mais plutôt une péninsule ${ }^{6}$. Au total, la synchronisation des pays de la région baltique avec ceux du reste de l'UE devrait coûter environ 1,2 milliard d'euros, dont $75 \%$ financés par l'Union, mais on note une montée en puissance de l'implication des pays concernés. C'est ainsi, par exemple, que l'Estonien Elering a annoncé en novembre 2019 son projet d'investir 111 millions d'euros dans la deuxième phase de synchronisation.

\footnotetext{
${ }^{6}$ Anke Schmidt-Felzmann, », in Māris Andžāns, Ilvija Bruğe (dir.), The Baltic Sea Region : Hard and Soft Security Reconsidered, Latvian Institute of International Affairs, Riga, 2016, p. 75.
} 
Le potentiel de croissance des EnR est en outre considéré comme important dans la région. Là aussi, des interconnexions sont à l'œuvre, comme par exemple entre la Pologne et le nord de l'Allemagne.

L'électricité nucléaire est l'objet de vastes débats. La Lituanie disposait d'une centrale, construite durant la période soviétique, fournissant $70 \%$ de l'électricité du pays et dotée de deux réacteurs puissants (1 $500 \mathrm{MW}$ chacun) mais de type RBMK, id est semblables à ceux de Tchernobyl. L'UE a donc fait de l'engagement de Vilnius à fermer cette centrale un préalable à l'adhésion de la Lituanie à l'Union. Les autorités lituaniennes ont ensuite décidé de construire une nouvelle centrale, à proximité de l'ancienne, en coopération avec le Japonais Hitachi $^{7}$. La centrale aurait pu alimenter en électricité les pays voisins, Estonie et Lettonie en particulier, la Pologne s'étant retirée du projet fin 2011 après avoir jugé la proposition lituanienne trop peu conforme à ses intérêts. Le projet, soutenu par l'UE, entrait également dans le PIMERB. Mais un référendum organisé dans le pays en octobre 2012 a mis fin aux espoirs des autorités lituaniennes, puisque la population a rejeté à $63 \%$ l'hypothèse d'une nouvelle centrale. C'est un coup dur porté à l'indépendance énergétique de la Lituanie : après la fermeture de la centrale d'Ignalina, le pays a dû importer $80 \%$ de son énergie de Russie. Une nouvelle centrale lui aurait permis de ne dépendre qu'à $35 \%$ environ des importations d'énergie.

Le retrait préalable de la Pologne, refusant de participer au projet au motif de la trop faible importance qui lui était accordée, mérite d'être souligné. La concurrence à l'œuvre dans la région a trouvé là un motif d'expression, Varsovie ne tolérant pas de se voir accorder une participation au projet à peu près équivalente à celle des autres partenaires, jugés plus « petits » par la Pologne. Cette susceptibilité polonaise est assez révélatrice des rapports entre pays de la région.

La multitude des projets de construction de centrales nucléaires est, elle aussi, emblématique des concurrences à l'œuvre : alors que Vilnius envisageait encore de construire la nouvelle centrale d'Ignalina, la Pologne laissait planer un doute sur ses propres intentions au regard du nucléaire. En 2012, l'autorité compétente autorisait le principe d'installation de deux centrales dans le pays, pour une capacité totale de 3000 MW. Le projet semble avoir été depuis abandonné. La Russie, quant à elle, se disait prête à lancer un projet dans l'enclave de Kaliningrad, avant d'y renoncer, peu après le référendum lituanien. L'Estonie, quant à elle,

\footnotetext{
${ }^{7}$ Le Russe Rosatom aurait souhaité participer au projet.
} 
s'interroge depuis 2019 sur l'opportunité de se doter d'une centrale nucléaire de taille modeste mais les autorités avancent avec prudence : si une telle centrale permettrait d'avancer sur la voie de sortie de l'exploitation des schistes bitumineux, elle risque néanmoins de se heurter à l'opposition de l'opinion publique. Pendant que les pays voisins hésitaient, la Biélorussie, elle, s'est lancée dans la construction d'une centrale à Astravets, malgré les protestations véhémentes de la Lituanie qui doute de la sûreté de cette installation distante de moins de $50 \mathrm{~km}$ de Vilnius. Dotée de deux réacteurs de 1200 MW chacun, elle est réalisée en coopération avec le Russe Rosatom. Elle a fait l'objet d'une campagne active de la part de Vilnius qui aurait espéré d'une part une surveillance accrue de cette installation par les instances européennes et suggère d'autre part avec insistance à l'ensemble des pays de la région de renoncer à l'achat d'électricité venant d'Astravets, seul moyen de disqualifier la centrale en rendant hypothétique sa rentabilité. Cet appel à la solidarité régionale peut passer par la non-synchronisation de la centrale biélorusse avec le réseau européen, ce qui suppose une approche cohérente des États. En 2016, les trois Baltes ont rédigé une déclaration commune affirmant leur volonté de faire barrage à l'entrée de toute forme d'énergie «non sûre » sur leurs territoires mais, à la veille de la mise en service du premier réacteur (prévue pour le premier trimestre 2020), seules la Lituanie et la Pologne s'étaient engagées à ne pas acheter d'électricité produite à Astravets. La Finlande, en revanche, ne semble pas vouloir se rallier à cette philosophie ; alors que Rosatom construit une centrale dans le nord-ouest du pays, à Pyhäjoki, Helsinki est peu enclin à compliquer ses relations avec son constructeur. Une dernière parade pour contrer la centrale d'Astravets consisterait à ne pas autoriser la Biélorussie à vendre sa production via le Nord Pool Spot, la bourse nordique de l'électricité.

Lancée en 2002, cette dernière atteste bien que la libéralisation du marché de l'électricité renforce l'efficacité des infrastructures dès lors que ce marché est concurrentiel. Actif dans neuf pays (Norvège, Suède, Finlande, Lituanie, Lettonie, Estonie, Danemark, Allemagne et Royaume-Uni), le Nord Pool constitue le premier marché dérégulé de l'électricité au monde et permet d'échanger plus de $80 \%$ de la consommation électrique du marché énergétique nordique et baltique.

\section{Le gaz, révélateur de solidarités inaccessibles?}

Jusque très récemment, les pays baltes et la Finlande importaient $100 \%$ de leur gaz de Russie ; la Pologne en importait $90 \%$. Pendant quelques années, cette situation a semblé satisfaisante à l'UE - dépendante en moyenne à $26 \%$ du gaz russe - et Bruxelles a activement promu le 
partenariat énergétique Russie-UE à partir de 2000. Les crises gazières de 2006 et 2009 ont changé la donne : les interruptions momentanées de livraisons de gaz russe via l’Ukraine provoquées par les différends entre les deux pays ont amené Bruxelles à remettre en cause ce qui est désormais considéré comme une « dépendance ». Aujourd'hui, le mot d'ordre est celui de la triple diversification : celle des sources d'énergie, celle des fournisseurs et celle des voies d'approvisionnements. On se méfie de la Russie, soupçonnée d'utiliser son atout énergétique comme une arme. Moscou, de son côté, affirme que c'est la Russie qui est la plus vulnérable du fait de sa dépendance vis-à-vis d'un client européen qu'elle juge instable (70\% de ses exportations d'hydrocarbures vont vers l'Europe).

En matière gazière, les décisions sont toutefois contraintes par le coût d'installation et d'entretien des infrastructures. Une fois posé, un tube doit pouvoir acheminer du gaz pendant plusieurs décennies. Pourtant, force est de constater depuis quelques années que la politique développée par la Russie en matière de voies de sortie de son gaz est radicale, même si coûteuse : l'objectif de Moscou est de diversifier ces voies afin de réduire sa dépendance visà-vis des pays de transit. L'Ukraine est tout particulièrement dans le viseur mais elle n'est pas la seule. C'est ainsi que la Russie développe depuis la fin des années 1990 des voies alternatives: alors que $90 \%$ du gaz russe destiné à l'Europe transitait par l'Ukraine jusqu'en 2000, les tubes Iamal (via la Biélorussie et la Pologne), Blue Stream (via la mer Noire vers la Turquie), Nord Stream (via la mer Baltique vers l'Allemagne) et, bientôt, TurkStream (via la mer Noire vers l'Europe balkanique) vont réduire le rôle de l'Ukraine en tant que pays de transit et répartir les volumes de gaz, laissant plus de marge de négociation à la Russie ${ }^{8}$.

\section{Diversifier les interconnexions}

Les pays baltiques se sont lancés, eux aussi, dans ce processus de triple diversification. On a vu que le mix énergétique des pays de la région est assez complexe et soumis à de larges changements. Le gaz reste toutefois, globalement, fondamental. Il s'agit donc de trouver de nouveaux fournisseurs, pour réduire le poids de la Russie, et de diversifier les voies. Des interconnexions sont en cours, se traduisant par l'accès à de nouveaux interlocuteurs et par la pose de nouveaux tubes. En outre, la montée en puissance du GNL apparaît comme une aubaine, puisqu'elle affranchit les pays consommateurs des contraintes d'installations

\footnotetext{
${ }^{8}$ Céline Bayou, « Russie - Europe : le complexe dossier du gaz », Politique internationale, $\mathrm{n}^{\circ} 163$, Printemps 2019.
} 
pérennes : une fois liquéfié, le gaz naturel est facilement transportable sur de longues distances et rend possible l'importation auprès de fournisseurs variés.

Concernant les nouvelles interconnexions, on peut citer le BalticConnector, interconnecteur gazier d'une capacité annuelle de 2,6 milliards de $\mathrm{m}^{3}$ mis en service en janvier 2020 et reliant la Finlande et l'Estonie. Financé à $75 \%$ par l'UE, il permet de transporter du gaz dans les deux sens. Il est utile au désenclavement de la Finlande qui a ainsi accès aux réseaux européens et peut en outre profiter des réservoirs naturels d'Inčukalns, situés en Lettonie, d'une capacité de 4,47 milliards de $\mathrm{m}^{3}$. Le paradoxe est que ce tube peut acheminer vers les pays baltes du gaz en provenance de Finlande, ce qui apparaît comme une avancée en matière de réduction de la dépendance gazière vis-à-vis de la Russie... à ceci près que le gaz vient bien de Russie. BalticConnector n'en est pas moins présenté comme un outil de diversification et donc de réduction de la menace qui pourrait contribuer en outre à la baisse du prix du gaz proposé par la Russie à la Finlande, concurrence oblige. Un autre gazoduc doit relier à horizon 2022 la Lituanie et la Pologne : le Gas Interconnection Poland Lithuania (GIPL) répond aux mêmes besoins. Il s'étendra sur $562 \mathrm{~km}$ et permettra de transporter 2,3 milliards de $\mathrm{m}^{3}$ de gaz par an. La Pologne envisage en outre l'installation du Northern Gate (ou Norwegian Corridor) qui doit lui permettre d'acheter 10 milliards $\mathrm{de}^{3} /$ an de gaz norvégien, combinant tubes terrestres et sous-marins, et transitant notamment par le Danemark. Soutenu par l'UE, le tube devrait être installé d'ici 2022. D'autres projets encore sont discutés : la modernisation du gazoduc Lettonie-Estonie afin de faire passer sa capacité de 7 à 10 millions de $\mathrm{m}^{3}$ par jour et d'y adjoindre une station de compression permettant de procéder à des flux inversés, celle du gazoduc Estonie (Narva)-Russie, dans les mêmes buts (capacité envisagée de 0,5 à 7,5 millions de $\mathrm{m}^{3}$ par jour), ou encore celle du gazoduc Lettonie-Lituanie, qui devrait passer de 5 à 12 millions de $\mathrm{m}^{3}$ par jour. Au final, les États baltes, la Finlande et la Pologne pourront donc échanger du gaz, dans un double sens, varier les voies de transport et aussi les fournisseurs. Ils seront dès lors mieux prémunis contre d'éventuelles ruptures d'approvisionnement. Et donc contre d'éventuelles pressions politiques.

\section{La bataille des terminaux de GNL}

Par ailleurs, les installations pour accueillir du gaz naturel liquéfié prolifèrent dans la région. L'option d'un terminal de GNL commun à la région a été sérieusement envisagée, donnant lieu à des tractations entre la Finlande, l'Estonie, la Lettonie et la Lituanie ${ }^{9}$. En 2013, la

\footnotetext{
${ }^{9}$ Pour plus de précision, voir Céline Bayou, « Région baltique : qui gagnera le terminal de gaz naturel liquéfié ? », Regard sur l'Est, 15 mai 2013.
} 
demande annuelle pour un tel terminal a été évaluée à 11 milliards de $\mathrm{m}^{3}$ de gaz. Mais les quatre protagonistes se sont avérés incapables d'élaborer une politique régionale cohérente, parvenant même à décourager 1'UE : elle était pourtant prête à financer en partie la construction du terminal, à condition que celui-ci soit régional précisément. La concurrence entre les acteurs s'est révélée d'autant plus rude qu'elle a mêlé intérêts d'affaires et fierté nationale. Les autorités lituaniennes ont été les premières à quitter le jeu, annonçant au printemps 2011 que la Lituanie louerait un tanker et l'utiliserait comme petit terminal flottant. La Lettonie, elle, a tenté de faire valoir son «droit » moral à se doter du terminal, puisque la Lituanie pouvait alors se targuer d'avoir bientôt une nouvelle centrale nucléaire et que l'Estonie prévoyait d'installer ses câbles Estlink. Il est vrai qu'un terminal letton aurait présenté en outre l'avantage de pouvoir bénéficier à courte distance des infrastructures de stockage d'Inčukalns. Argument vite écarté par les Estoniens, qui ont rappelé que ces réservoirs sont pour le moment sous contrat exclusif avec... Gazprom et que le tube qui relie les réservoirs à leur propre pays serait certes suffisant pour approvisionner leur marché mais pas pour fournir ensuite le marché finlandais. À cet égard, ont-ils noté, le terminal aurait tout à gagner à être installé dans leur pays. La Finlande est aussi entrée dans la course, faisant valoir sa capacité à créer les conditions d'une véritable concurrence industrielle et ses besoins importants en volume. La question de l'actionnariat des acteurs de cette négociation a aussi joué un rôle, l'idée étant que le futur terminal ne soit pas «pollué » par la Russie. Or, par exemple, le principal actionnaire de l'opérateur estonien, Eesti Gaas, n'était autre que Gazprom. Finalement, le projet d'un terminal commun a échoué, et l'UE n'a pu soutenir aucun projet régional.

En 2014, la Lituanie a inauguré son terminal flottant à Klaipeda, opportunément baptisé «Independence » et doté d'une capacité de 4 milliards de $\mathrm{m}^{3}$ de gaz par an. Celle-ci est suffisante pour couvrir les besoins du pays et exporter le surplus vers l'Estonie et la Lettonie lorsqu'elles le souhaitent. Puis, en 2016, la Pologne a également inauguré son terminal de GNL, à Świnoujście, doté d'une capacité de 5 milliards de $\mathrm{m}^{3} / \mathrm{an}$. Toujours en 2016 , la Finlande a elle aussi inauguré un petit terminal de GNL à Pori, exclusivement destiné à alimenter l'industrie maritime, et annoncé son intention d'en construire d'autres, à Tornio, Hamina et Rauma.

À mesure que ces terminaux sont disséminés dans la région, ils tuent l'idée d'une installation de grande taille, qui se serait avérée sans doute plus économique et aurait mutualisé les risques. Certes, sous l'effet de cette politique volontariste, la Lituanie a vu, à sa propre surprise, la 
Russie lui concéder des prix de livraison du gaz plus avantageux que précédemment. Le GNL norvégien ou états-unien qu'elle importe lui coûte plus cher que les fournitures traditionnelles de gaz russe, par tubes ; il est plus cher également que le GNL que la Russie lui propose aussi désormais. C'est ainsi qu'en 2019, plusieurs livraisons de GNL russe sont arrivées jusqu'au terminal Indépendance, dans une relative discrétion. Les pays n'en restent pas moins enthousiastes : à l'automne 2016, les autorités polonaises avaient plaidé auprès du Parlement européen l'option du projet Porte gazière du Nord, basé sur le doublement de la capacité du terminal de Świnoujście et une interconnexion rapide par tubes avec le Danemark et la Norvège (Northern Gate), faisant valoir qu'elle pourrait ensuite alimenter en gaz l'Europe centrale et orientale. Cette stratégie, dont la rentabilité doit encore être démontrée, a beau être présentée comme coopérative, elle met surtout en avant les intérêts de la seule Pologne, sous couvert de diversification et donc d'accroissement de la sécurité énergétique régionale. La méthode, elle, n'est pas régionale et ce constat d'échec coûte cher à l'ensemble des pays.

\section{Nord Stream : la solidarité comme Graal, l'individualisme comme quotidien}

Le gazoduc Nord Stream montre, lui aussi, que la fin de l'isolement énergétique de la région baltique ne peut s'empêcher de passer par le triomphe d'intérêts particulier. Négocié entre le chancelier allemand Gerhard Schröder et le président russe Vladimir Poutine, il achemine du gaz russe sur $1224 \mathrm{~km}$ depuis le fond du golfe de Finlande jusqu'en Allemagne via la mer Baltique, en évitant les pays de transit. Lors de l'annonce du projet, à la fin de 2005, la Pologne et les États baltes ont protesté contre cet accord signé « par-dessus [leurs] têtes » et clairement à leur désavantage. Ces nouveaux États membres de l'UE ont alors dénoncé le manque de solidarité européenne et tenté de s'opposer au projet. En vain. En septembre 2011, le gazoduc a été inauguré, doté d'une capacité annuelle de 55 milliards de $\mathrm{m}^{3}$ de gaz. Outre le fait qu'il ignorait les intérêts d'un certain nombre de pays fortement dépendants du gaz russe et qui auraient vu un intérêt à faire passer un tel tube sur leur territoire afin de bénéficier de recettes de transit et d'amoindrir ainsi leur facture énergétique, ce tube semblait bien aller à l'encontre de la politique énergétique commune que l'UE peine tant à mettre en œuvre. Il contredit en effet les principes de diversification des fournisseurs, renforçant au contraire la dépendance européenne vis-à-vis du gaz russe. Il contredit également les engagements de l'UE qui, tout en affirmant souhaiter soutenir l'économie de l'Ukraine, joue ainsi le jeu russe de diversification des voies d'exportation ${ }^{10}$. Il viole aussi un des règlements européens adopté

\footnotetext{
${ }^{10}$ Céline Bayou, « Ukraine/Russie. Dimension gazière d'un conflit », Note $\mathrm{n}^{\circ} 16$, Fondation pour la Recherche Stratégique, 16 juillet 2015 (http://www.frstrategie.org/publications/notes/web/documents/2015/201516.pdf).
} 
en 2011, qui impose d'une part la séparation (unbundling) entre producteur, transporteur et distributeur de gaz et d'électricité, et garantit d'autre part l'accès aux tiers des tubes traversant l'Europe. L'UE est en mesure de faire appliquer ce règlement (appelé $3^{\mathrm{e}}$ Paquet énergétique) et c'est bien grâce à lui qu'elle a forcé la Russie à abandonner, fin 2014, le projet de gazoduc South Stream qui devait traverser la mer Noire puis les Balkans pour rejoindre l'Autriche ${ }^{11}$. S'agissant du gazoduc allemand Opal qui est relié au Nord Stream et qui longe la frontière orientale de l'Allemagne jusqu'en République tchèque, depuis son lancement en 2011, celuici a été exempté des règles du marché intérieur sur l'accès des tiers au réseau. Ce n'est que fin octobre 2016 que la Commission a décidé que, dorénavant, l'utilisation de $50 \%$ seulement de la capacité d'Opal serait exemptée de ces règles, le reste devant s'y conformer. La décision a été jugée par nombre de pays comme le triomphe de Gazprom.

L'annonce, en juin 2015, du projet de doublement du Nord Stream n'a pas apaisé la situation. Aux deux conduites initiales vont s'ajouter deux autres tubes, plus ou moins parallèles, faisant passer la capacité totale des Nord Stream 1 et 2 à 110 milliards de $\mathrm{m}^{3} / \mathrm{an}$. Présenté par la chancelière allemande Angela Merkel et le président russe V. Poutine comme un projet mû par des intérêts purement économiques, il a amené l'ex-vice-président de la Commission européenne en charge de l'Union de l'énergie, Maroš Šefčovič, à ironiser : «Je n'ai jamais vu des projets commerciaux discutés aussi souvent et à un tel niveau politique », a-t-il noté, avant d'ajouter qu'il serait particulièrement attentif à la conformité du gazoduc avec les règles de l'UE. De toute évidence, l'Allemagne ne l'entendait pas ainsi et Sigmar Gabriel, alors ministre fédéral de l'Économie et de l'Énergie, a rapidement fait savoir qu'il souhaiterait que ce tube reste sous la compétence légale des autorités allemandes.

Face à ce qu'ils ont perçu comme une nouvelle trahison, certains pays de la région baltique ont décidé de contre-attaquer. La Pologne a été la plus active sur ce terrain, forçant les partenaires européens du Nord Stream $2^{12}$ à renoncer en août 2016 à créer une société mixte autour de ce projet, à la suite de l'avis défavorable rendu par l'autorité polonaise de la concurrence : déjà présentes sur le marché polonais, ces entreprises auraient mis en cause, avec le Nord Stream 2, le niveau de concurrence dans le secteur par leur position dominante. En décembre 2016, la Pologne a porté un nouveau coup au projet, en déposant une plainte par le biais de la filiale allemande du monopole polonais du gaz, PGNiG Supply \& Trading GmbH,

\footnotetext{
${ }^{11}$ Céline Bayou, « Turkish Stream : la bataille ne fait que commencer », Diploweb, 9 juin 2015.

${ }^{12}$ Les Allemands Uniper et Wintershall, le Français Engie, l'Autrichien OMV et l'Anglo-néerlandais Royal Dutch/Shell.
} 
contre la décision d'exemption partielle d'Opal des règles communes européennes. L'Ukraine, qui pourrait se trouver deux fois victime du Nord Stream, soutient activement les démarches polonaises. Pourtant, le 24 avril 2017, Gazprom et ses cinq partenaires européens ont signé un accord de financement aux termes duquel la société russe est restée le seul actionnaire du projet (ses partenaires le finançant en partie). La structure de financement répond donc aux objections des autorités de la concurrence polonaise.

Les pays baltes, pas moins opposés au projet, sont restés plus discrets, partant du constat de leur relative impuissance à s'opposer frontalement à la fois à la Russie et à l'Allemagne. Ils ont préféré s'inscrire dans une démarche centre-orientale, d'autres pays de la région - notamment la République tchèque - ayant fait part de leurs réticences face à ce montage. Les pays nordiques, eux aussi, sont partagés ${ }^{13}$ : leur principal angle d'attaque a été le respect du $3^{\mathrm{e}}$ Paquet énergétique, même si l'expérience leur montre que l'argument est fragile. Eux aussi jugent risqué d'entrer en conflit avec l'Allemagne et avec la Russie, alors que le contexte sécuritaire en mer Baltique s'est fortement détérioré depuis 2014 et l'annexion de la Crimée par la Russie. Pour ces mêmes raisons sécuritaires, la Suède a hésité à autoriser la construction d'une plateforme de services près de Gotland, au moment même où Stockholm venait de décider de remilitariser son île. La Suède a aussi balancé (avant de renoncer) entre l'intérêt économique à voir ses ports de Slite (Gotland) et Karlshamn (sud du pays) stocker des tubes durant la construction et le risque de sabotage qu'une telle décision aurait fait peser sur son territoire, une présence physique russe ne l'enchantant que faiblement. La Finlande, plus attentiste quand il s'agit des intérêts du voisin russe, a peu fait entendre sa voix. C'est le Danemark $^{14}$ qui, tout en arguant d'interrogations exclusivement environnementales, aura finalement le plus contribué à retarder le projet : l'autorisation de passage du tube au sud de l'île de Bornholm (id est à proximité de Nord Stream 1) n'a été accordée que le 30 octobre 2019, après des mois d'atermoiements ${ }^{15}$. Nord Stream 2, qui devait être mis en service en janvier 2020, ne le sera, au plus tôt qu'au printemps ou à l'automne suivants. Ce retard est lourd de conséquences : alors que le contrat de transit gazier qui liait la Russie et l'Ukraine arrivait à expiration le 31 décembre 2019, les retard cumulés de Nord Stream 2 et, plus au Sud,

\footnotetext{
${ }^{13}$ Justyna Gotkowska, Piotr Szymański, « The Nordic countries on Nord Stream 2 : between scepticism and neutrality », OSW Commentary, n 223,12 octobre 2016.

${ }^{14}$ Mihail Krutihin, « Nepokorennaâ Evropa : kakuû cenu pridëtsâ zaplatit' Rossii za 'Severnyj potok 2 ? » (Europe inconquise : quel prix la Russie devra-t-elle payer pour Nord Stream 2 », Forbes, 4 novembre 2019. ${ }^{15}$ Copenhague a d'abord envisagé de faire passer le tube au nord de l'île mais y a renoncé en raison de la présence de la fosse de Bornholm, fortement polluée. Elle a ensuite évoqué un passage très au Sud mais la délimitation des eaux territoriales avec la Pologne, intervenue entretemps, a placé ce tracé en Pologne. La décision prise à l'automne 2019 est finalement celle qui, d'emblée, aurait semblé la plus logique et simple.
} 
de TurkStream, retardent aussi le moment où Moscou pourra considérer que la Russie peut, si elle le souhaite, se passer totalement de la voie ukrainienne. La difficile négociation du futur contrat gazier russo-ukrainien en a subi les conséquences, Kiev négociant un accord de transit de dix ans, tandis que Moscou tentait d'arracher un contrat d'un an, le temps d'achever ses gazoducs de contournement.

Tous les pays de la région et au-delà ${ }^{16}$ sont bien conscients des enjeux : une fois construit, le Nord Stream 2 achèvera de marginaliser l'Ukraine sur la carte européenne, accroîtra la dépendance de l'Allemagne vis-à-vis du gaz russe mais augmentera aussi son poids en tant que pays redistributeur en Europe et il contribuera à affaiblir une politique énergétique européenne qui peine à émerger.

La Russie use-t-elle dans la région de la mer Baltique de cette fameuse « arme énergétique » dont certains lui font parfois grief ? S'il est en effet clairement de son intérêt de retarder la déconnexion/reconnexion énergétique de certains des États baltiques, cette politique qualifiée $\mathrm{d}$ 'arme des faibles semble pourtant désormais obsolète à certains ${ }^{17}$ : les moyens de pression énergétique de la Russie auraient été rendus inopérants par les changements survenus sur les marchés mondiaux et européens de l'énergie, qui ont accru la marge de manœuvre des pays consommateurs. La pression directe serait donc d'une efficacité moins importante qu'auparavant face, notamment, aux souplesses offertes par les hydrocarbures non conventionnels, par la montée du GNL et des EnR ou par les fluctuations des cours des hydrocarbures.

Dans la région baltique, force est toutefois de constater que c'est le manque de coopération qui laisse un champ certain à l'influence russe et lui permet parfois d'imposer ses conditions. Dans cette confrontation d'intérêts contraires, la difficulté des acteurs - qu'il s'agisse des États, des institutions ou des entreprises - à élaborer une politique cohérente et solidaire constitue sans doute la plus grande faiblesse d'une région qui prône pourtant l'approche coopérative. Finalement, la difficulté à mettre en œuvre un plan détaillé d'interconnexions énergétiques, à sacrifier certains intérêts nationaux à une cause commune pourtant unanimement perçue comme essentielle en termes sécuritaires, mais aussi la volatilité de l'UE

\footnotetext{
${ }^{16}$ Le rôle des États-Unis doit être signalé, avec le vote le 11 décembre 2019 d'un projet de loi imposant des sanctions aux entreprises participant à l'installation de Nord Stream 2, officiellement en soutien à l'Ukraine, mais visiblement aussi en soutien aux exportations américaines de GNL vers l'Europe.

${ }^{17}$ Pavel Baev, « La Russie et l'Europe centrale et orientale : entre confrontations et connivences », Notes de l'IFRI, n 97 , novembre 2016, p. 29.
} 
ou de certains de ses États membres semblent bien être les véritables menaces qui pèsent sur cette région. Menaces qui ont ceci de caractéristique qu'elles évitent à la Russie d'avoir à déployer une politique particulièrement agressive en matière énergétique. 